\title{
Existence of weak solutions in elasticity
}

\author{
Victor A Eremeyev \\ Faculty of Mechanical Engineering, Otto von Guericke University Magdeburg, Germany; Department of Math- \\ ematics, Mechanics and Computer Science, Southern Federal University and Laboratory of Smart Materials \\ of Southern Scientific Centre of RASci, Russia
}

\section{Leonid P Lebedev}

Departamento de Matemáticas, National University of Colombia, Colombia

\begin{abstract}
Solvability and uniqueness of solutions to the problems of equilibrium, vibration and dynamics in a weak setup for classical and nonclassical models of linear elasticity are established in a unified framework sufficiently flexible to accommodate new elastic models.
\end{abstract}

\section{Keywords}

Elasticity, weak solution, generalized solution, existence and uniqueness of solution

\section{Introduction}

Being convinced that the perfect world should be described in a perfect manner — optimally — the old mechanicists such as L. Euler and D. Bernoulli sought integral extremal principles for various natural objects. They established principles relating the solution of boundary-value problems in mechanics with the location of extremals of certain functionals, and in this way, arrived at the notion of energy. Together with Newtonian dynamics, there also appeared Lagrangian mechanics, based on variational ideology.

Up to a certain point, the description of various models in classical and continuum mechanics was carried out using systems of differential equations supplemented with boundary conditions. Conservation laws and variational principles stood as important but adjunct parts of the theory, although some differential equations were deduced from variational principles. A change came with the widespread use of computers and finite-element methods (FEMs) whose equations were derived directly from mechanical variational methods. FEM solutions turned out to be approximations to the so-called weak solutions of mechanical boundary-value problems, which were determined by setting the first variation of a certain functional to zero for all admissible disturbances of the solution.

For equilibrium problems in elasticity, weak solutions are determined by Lagrange's variational principle. This leads to the equations of the virtual work principle, which can be considered as independent of Lagrange's principle and, moreover, is more general than the latter. For dynamic problems, weak solutions were introduced via a modification of the Gauss principle. 
Theoretical studies of generalized (weak) solutions to boundary-value problems were initiated by S.L. Sobolev in relation to a problem in hydromechanics. His results on the so-called Sobolev spaces, subsequently collected in Sobolev [1], became instrumental to the theory of partial differential equations. The introduction of generalized solutions was originally based on variational principles.

In linear elasticity, the decisive step facilitating the study of weak solutions was taken by Korn [2, 3] well before their introduction. Korn established an inequality pertaining to the smooth displacement vector $\mathbf{u}$ for an elastic body occupying a bounded volume $V \subset \mathbb{R}^{3}$ with some part $\partial V_{1}$ of the boundary $\partial V$ rigidly fixed (clamped)

$$
\left.\mathbf{u}\right|_{\partial V_{1}}=\mathbf{0} .
$$

Korn's inequality has the form

$$
\int_{V} \mathbf{u}^{2} d V \leq c\|\mathbf{u}\|_{E}^{2}, \quad\|\mathbf{u}\|_{E}^{2}=\int_{V} c^{i j k l} \varepsilon_{i j} \varepsilon_{k l} d V
$$

where $\varepsilon_{i j}=\frac{1}{2}\left(\partial u_{i} / \partial x_{j}+\partial u_{j} / \partial x_{i}\right)$ are the Cartesian components of the strain tensor $\epsilon$. Here and in what follows, we use the notation $\mathbf{u}^{2}=\mathbf{u} \cdot \mathbf{u}$ for any vector $\mathbf{u}$. The components $\left(c^{i j k l}\right)$ of the tensor of elastic moduli possess the properties of symmetry and positive definiteness typical in elasticity

$$
c^{i j k l} \varepsilon_{i j} \varepsilon_{k l} \geq c_{0} \varepsilon_{i j} \varepsilon_{i j} .
$$

Moreover, the positive constant $c_{0}$ is independent of $\left(\varepsilon_{i j}\right)$, and the positive constant $c$ is independent of $\mathbf{u}$. Here, we assume Einstein's summation rule over repeated indices. In what follows, the notation $c_{k}$ will be used to signify positive constants that are independent of the set of functions over which inequalities are established.

If no portion of $\partial V$ is clamped, then (2) fails to hold for so-called small rigid displacements of the form $\mathbf{u}_{r}=\mathbf{a}+\mathbf{b} \times \mathbf{r}$, where $\mathbf{a}, \mathbf{b}$ are arbitrary vector constants and $\mathbf{r}$ is the position vector such that $\boldsymbol{\epsilon}\left(\mathbf{u}_{r}\right)=\mathbf{0}$. In this case, an extension of Korn's inequality, similar to H. Poincaré's inequality, is valid

$$
\int_{V} \mathbf{u}^{2} d V \leq c_{1}\left\{\left(\int_{V} \mathbf{u} d V\right)^{2}+\left(\int_{V} \mathbf{r} \times \mathbf{u} d V\right)^{2}+\|\mathbf{u}\|_{E}^{2}\right\} .
$$

These inequalities are extended to inequalities in the Sobolev space $\left(W^{1,2}(V)\right)^{3}$, where the norm is

$$
\|\mathbf{u}\|_{W}^{2}=\int_{v}\left(\mathbf{u}^{2}+\left(\frac{\partial \mathbf{u}}{\partial x_{1}}\right)^{2}+\left(\frac{\partial \mathbf{u}}{\partial x_{2}}\right)^{2}+\left(\frac{\partial \mathbf{u}}{\partial x_{3}}\right)^{2}\right) d V
$$

For vector functions $\mathbf{u} \in\left(W^{1,2}(V)\right)^{3}$ satisfying (1), Korn's inequality becomes

$$
\|\mathbf{u}\|_{W} \leq c_{2}\|\mathbf{u}\|_{E}
$$

and for a free body, we have

$$
\|\mathbf{u}\|_{W} \leq c_{3}\left\{\left(\int_{V} \mathbf{u} d V\right)^{2}+\left(\int_{V} \mathbf{r} \times \mathbf{u} d V\right)^{2}+\|\mathbf{u}\|_{E}^{2}\right\}^{1 / 2}
$$

for all $\mathbf{u} \in\left(W^{1,2}(V)\right)^{3}$.

These two inequalities and their backwards versions (which are trivial) state that the expressions on the righthand sides of (3) and (4) can serve as equivalent norms in corresponding subspaces of the space $\left(W^{1,2}(V)\right)^{3}$, and thereby permit use of the Sobolev imbedding theorem. This allows us to introduce rigorous definitions of weak generalized solutions for the various problems of static and dynamic elasticity.

In this paper, we analyze initial and boundary-value problems of linear elasticity, in particular, for bodies that are elastically supported on some portions of their boundaries. As a support model, we take the boundary condition under which the stress vector is proportional to the boundary displacement. A particular case of an elastic support is Winkler's foundation. We also consider problems describing surface elastic effects within the 
framework of the Gurtin-Murdoch model [4]. From a physical standpoint, the model can be regarded as an elastic body with a membrane (film) glued to some portion of its boundary. The membrane model generalizes the notion of liquid surface tension to the case of an elastic solid. In Steigmann and Ogden [5], the equations of Gurtin and Murdoch [4] were extended to describe the bending stiffness of the attached elastic film; in Javili and Steinmann [6], they were generalized to thermoelasticity. In recent decades, the model of Gurtin and Murdoch [4] has been used in nanomechanics [7, 8] and in fracture problems [9, 10]. Some mathematical properties of the initial and boundary-value problems in surface elasticity, discovered through various methods, can be found in Schiavone and $\mathrm{Ru}$ [11], Altenbach et al. [12, 13] and Javili et al. [14].

It should be noted that considerations of mathematical dynamic elasticity are presented for Dirichlet boundary conditions or for certain mixed conditions [15-17] when some portion of the boundary is fixed. For equilibrium problems, when the boundary is not geometrically constrained and hence the load is given on the boundary, the necessary condition for solvability is that the load be self-balanced. Here, we also consider dynamic problems for a body free of geometric constraints on the boundary; solvability is established under certain smoothness conditions for the load.

\section{Static problems of elasticity}

The results for classical elasticity are well known [15-17], but for the sake of completeness, we present a brief summary. We begin with the concept of energy space.

Definition 2.1. The space $E_{0}$ is the completion, with respect to the norm $\|\cdot\|_{E}$, of the set of vector functions $\mathbf{u}$ that are continuously differentiable on $\bar{V}$ and satisfy (1).

The norm $\|\cdot\|_{E}$ naturally induces the inner product

$$
(\mathbf{u}, \mathbf{v})_{E}=\int_{V} c^{i j k l} \varepsilon_{i j}(\mathbf{u}) \varepsilon_{k l}(\mathbf{v}) d V
$$

so $E_{0}$ is a Hilbert space. The argument $\mathbf{u}$ in $\varepsilon_{i j}(\mathbf{u})$ denotes the displacement vector for which $\varepsilon_{i j}$ is calculated.

As stated above, on the subspace of $\left(W^{1,2}(V)\right)^{3}$ consisting of the vector functions satisfying (1), the norms $\|\cdot\|_{E}$ and $\|\cdot\|_{W}$ are equivalent, and we can use Sobolev's imbedding theorem formulated for $W^{1,2}(V)$.

The total strain energy for an elastic body is

$$
\mathcal{E}(\mathbf{u})=\frac{1}{2}(\mathbf{u}, \mathbf{u})_{E}-\int_{V} \mathbf{F} \cdot \mathbf{u} d V-\int_{\partial V_{2}} \mathbf{f} \cdot \mathbf{u} d S,
$$

where $\mathbf{F}$ represents given volume forces and $\mathbf{f}$ represents surface forces distributed over $\partial V_{2}=\partial V \backslash \partial V_{1}$. The terms

$$
\mathcal{W}(u)=\int_{V} \mathbf{F} \cdot \mathbf{u} d V+\int_{\partial V_{2}} \mathbf{f} \cdot \mathbf{u} d S
$$

represent the work of external forces acting on $V$ over the displacement u. Using Lagrange's variational principle, we can introduce a weak (or energy, or generalized) solution to the equilibrium problem.

Definition 2.2. The element $\mathbf{u} \in E_{0}$ is a weak (energy) solution of the equilibrium problem for the body occupying $V$ with external forces $(\mathbf{F}, \mathbf{f})$ if it minimizes $\mathcal{E}(\mathbf{u})$ on the space $E_{0}$.

As a minimum point of $\mathcal{E}(\mathbf{u})$, the vector function $\mathbf{u}$ satisfies the integro-differential equality

$$
\left.\frac{d}{d t} \mathcal{E}(\mathbf{u}+t \mathbf{v})\right|_{t=0}=0,
$$

for any fixed $\mathbf{v} \in E_{0}$. This equality, which in the calculus of variations is called the equality of the first variation of the functional to zero, now takes the form

$$
(\mathbf{u}, \mathbf{v})_{E}-\int_{V} \mathbf{F} \cdot \mathbf{v} d V-\int_{\partial V_{2}} \mathbf{f} \cdot \mathbf{v} d S=0 .
$$


Holding for any $\mathbf{v} \in E_{0}$, it defines a weak/energy solution to the equilibrium problem and permits us to formulate various versions of the FEM for elasticity problems.

Using standard tools of the calculus of variations, from (5), we can deduce the equilibrium equations of elasticity and the natural boundary conditions over $\partial V_{2}$. The latter express the equality of the corresponding tension to $\mathbf{f}$ on $\partial V_{2}$. We do not present these relations, which can be found in any textbook on elasticity. Although standard tools in the theory of Sobolev spaces allow us to study the regularity of a weak solution, assuming some degree of regularity for the load and smoothness of the boundary contour, we do not pursue such issues here.

Now we establish the following.

Theorem 2.1. A weak solution $\mathbf{u} \in E_{0}$ to equation (5) exists and is unique if the Cartesian components $\left(F_{1}, F_{2}, F_{3}\right)$ of $\mathbf{F}$ belong to the space $L^{6 / 5}(V)$ and the Cartesian components $\left(f_{1}, f_{2}, f_{3}\right)$ of $\mathbf{f}$ belong to the space $L^{4 / 3}\left(\partial V_{2}\right)$.

Note that the theorem is valid for $V$ satisfying the standard conditions under which Sobolev's imbedding theorem holds, i.e. $V$ is a connected bounded domain with a regular boundary satisfying the cone condition, which means there is a finite circular cone, the vertex of which can touch any point of $\partial V$ in such a way that the cone lies inside $V$.

The proof of Theorem 2.1 follows from Theorem 2.2.

Theorem 2.2. (Riesz representation theorem). For a linear bounded functional $F$ in a Hilbert space $H$, there exists a unique element $f \in H$ such that $F x=(x, f)_{H}$ for any $x \in H$, and $\|F\|=\|f\|_{H}$.

Indeed, $E_{0}$ is a Hilbert space and the work of external forces $\mathcal{W}(\mathbf{v})$ is a linear functional. By Sobolev's imbedding theorem, which guarantees that the imbedding operators from $W^{1,2}(V)$ to $L^{6}(V)$ and $L^{4}\left(\partial V_{2}\right)$ are continuous, and Hölder's inequality, we get

$$
|\mathcal{W}(\mathbf{v})| \leq\|\mathbf{F}\|_{\left(L^{6 / 5}(V)\right)^{3}}\|\mathbf{v}\|_{\left(L^{6}(V)\right)^{3}}+\|\mathbf{f}\|_{\left(L^{4 / 3}\left(\partial V_{2}\right)\right)^{3}}\|\mathbf{v}\|_{\left(L^{4}\left(\partial V_{2}\right)\right)^{3}} \leq c_{4}\|\mathbf{v}\|_{E} .
$$

Since the functional $\mathcal{W}$ is bounded, it can be represented as $\mathcal{W}(\mathbf{v})=\left(\mathbf{v}, \mathbf{u}^{*}\right)_{e}$ with a uniquely defined $\mathbf{u}^{*} \in E_{0}$. Hence, (5) can be written in the form

$$
(\mathbf{u}, \mathbf{v})_{E}-\left(\mathbf{u}^{*}, \mathbf{v}\right)_{E}=0 .
$$

Because this equation holds for any $\mathbf{v} \in E_{0}$, the weak solution $\mathbf{u}^{*} \in E_{0}$ exists and is unique.

\section{I. Equilibrium of a free elastic body}

Here we consider the equilibrium problem for an elastic body not subjected to geometric constraints. Now the work of external forces is

$$
\mathcal{W}_{0}(\mathbf{u})=\int_{V} \mathbf{F} \cdot \mathbf{u} d V+\int_{\partial V} \mathbf{f} \cdot \mathbf{u} d S .
$$

As we neglect the inertia terms, the minimum of total potential energy exists if and only if $\mathcal{E}\left(\mathbf{u}_{r}\right)=\frac{1}{2}\left(\mathbf{u}_{r}, \mathbf{u}_{r}\right)_{E}-$ $\mathcal{W}_{0}\left(\mathbf{u}_{r}\right)=0$ for any small rigid displacement $\mathbf{u}_{r}=\mathbf{a}+\mathbf{r} \times \mathbf{b}$. This means that $\mathcal{W}_{0}\left(\mathbf{u}_{r}\right)=0$ for any $\mathbf{u}_{r}$, hence for the existence of a minimum point of total potential energy, the load must be self-balanced. As $\mathbf{a}, \mathbf{b}$ are arbitrary vectors, this takes the usual form of load self-balance for a rigid body occupying volume $V$,

$$
\int_{V} \mathbf{F} d V+\int_{\partial V} \mathbf{f} d S=\mathbf{0}, \quad \int_{\mathbf{V}} \mathbf{r} \times \mathbf{F} \mathbf{d} \mathbf{V}+\int_{\partial \mathbf{V}} \mathbf{r} \times \mathbf{f} \mathbf{d} \mathbf{S}=\mathbf{0} .
$$

These relations, together with some regularity of the load, are sufficient to prove the following.

Theorem 2.3. Suppose the external load (F,f) is self-balanced in the sense of (7), and suppose the Cartesian components of $\mathbf{F}$ belong to $L^{6 / 5}(V)$, while those of $\mathbf{f}$ belong to $L^{4 / 3}(\partial V)$. There exists a weak solution $\mathbf{u}$ to the equilibrium problem for an unconstrained elastic body that minimizes the total potential energy functional $\mathcal{E}(\mathbf{u})=\frac{1}{2}(\mathbf{u}, \mathbf{u})_{E}-\mathcal{W}_{0}(\mathbf{u})$ in $\left(W^{1,2}(V)\right)^{3}$ and satisfies $(5)$ for any $\mathbf{v} \in\left(W^{1,2}(V)\right)^{3}$. The solution $\mathbf{u}$ is unique up to an arbitrary rigid displacement $\mathbf{u}_{r}=\mathbf{a}+\mathbf{b} \times \mathbf{r}$, and it has the form $\mathbf{u}^{*}+\mathbf{u}_{r}$. 
Any element $\tilde{\mathbf{u}} \in\left(W^{1,2}(V)\right)^{3}$ can be uniquely represented in the form $\tilde{\mathbf{u}}=\mathbf{u}+\mathbf{u}_{r}$, where $\mathbf{u}_{r}=\mathbf{a}+\mathbf{b} \times \mathbf{r}$ for constants $\mathbf{a}, \mathbf{b}$ and $\mathbf{u}$ satisfies the restrictions

$$
\int_{V} \mathbf{u} d V=\mathbf{0}, \quad \int_{\mathbf{V}} \mathbf{r} \times \mathbf{u} \mathbf{d} \mathbf{V}=\mathbf{0} .
$$

Let us denote the subspace of the elements of $\left(W^{1,2}(V)\right)^{3}$ satisfying (8) by $E_{f}$. In the equilibrium problem for a free elastic body occupying volume $V$, equation (5) is still valid (with $\partial V_{2}$ changed to $\partial V$ ) as it is a consequence of Lagrange's minimum energy principle. Taking a virtual displacement $\tilde{\mathbf{v}}=\mathbf{v}+\mathbf{v}_{r}$, where $\mathbf{v} \in E_{f}$ and $\mathbf{v}_{r}=\mathbf{a}_{1}+\mathbf{b}_{1} \times \mathbf{r}$, and substituting this into the transformed (5) while taking into account (7), we get an equation that does not contain $\mathbf{u}_{r}$ or $\mathbf{v}_{r}$,

$$
(\mathbf{u}, \mathbf{v})_{E}-\int_{V} \mathbf{F} \cdot \mathbf{v} d V-\int_{\partial V} \mathbf{f} \cdot \mathbf{v} d S=0 .
$$

This equation shares the same form as (5), and $\mathbf{u} \in E_{f}$ is its solution if (9) holds for any $\mathbf{v} \in E_{f}$. By (4), for any $\mathbf{v} \in E_{f}$, we get

$$
\|\mathbf{v}\|_{W} \leq c_{3}\|\mathbf{v}\|_{E}
$$

and so on $E_{f}$, a subspace of $\left(W^{1,2}(V)\right)^{3}$, the norms $\|\cdot\|_{E}$ and $\|\cdot\|_{W}$ are equivalent. Now for $\mathcal{W}_{0}$, we can mimic the estimates (6), obtaining $\mathcal{W}_{0}(\mathbf{v}) \leq c_{5}\|\mathbf{v}\|_{E}$ and concluding that $\mathcal{W}_{0}(\mathbf{v})$ is a bounded linear functional in $E_{f}$. So there is a unique element $\mathbf{u}^{*} \in E_{f}$ such that $\mathcal{W}_{0}(\mathbf{v})=\left(\mathbf{v}, \mathbf{u}^{*}\right)_{E}$, and our auxiliary problem (9) has the unique solution $\mathbf{u}^{*}$. For any $\mathbf{u}_{r}$, the sum $\mathbf{u}^{*}+\mathbf{u}_{r}$ satisfies (8) for any $\mathbf{v} \in\left(W^{1,2}(V)\right)^{3}$, and this completes the proof of Theorem 2.3.

Similarly, we can study the equilibrium problems for other boundary conditions when the rigid displacements of the elastic body exist but the dimension of the rigid displacement set is less than six. For example, we can consider the equilibrium problem for an elastic circular cylinder with the boundary constraint $\left.u_{n}\right|_{\partial V}=0$, $u_{n}=\mathbf{u} \cdot \mathbf{n}$, and given tangential forces on the boundary. The cylinder can rotate freely about its axis, and for the solvability of the corresponding equilibrium problem, aside from the restriction on regularity of the loads, the forces should be subjected to the self-balance condition for the rotational degree of freedom.

\subsection{Equilibrium of supported elastic bodies}

We can also consider equilibrium problems for elastic bodies with various types of elastic support, when the resistance of the external support is described by a simplified elastic model. Suppose that on some part $\partial V_{3}$ of the boundary, the tension vector $\mathbf{t}=\boldsymbol{\sigma} \cdot \mathbf{n}$, where $\sigma$ is the stress tensor, is given: $\left.\mathbf{t}\right|_{\partial V_{3}}=-k \mathbf{u}$, where $k>0$ is the support rigidity, a constant for simplicity. Suppose also that on $\partial V_{4}$, we have a Winkler-type support

$$
\left.\mathbf{t} \cdot \mathbf{n}\right|_{\partial V_{4}}=-\left.k^{\prime} \mathbf{u} \cdot \mathbf{n}\right|_{\partial V_{4}}=-\left.k^{\prime} u_{n}\right|_{\partial V_{4}},
$$

where $k^{\prime}>0$ is the rigidity. This problem can be formulated as a minimization problem for the energy functional, but to the elastic energy of the body, we should add the strain energy of the support. So the energy functional becomes

$$
\mathcal{E}_{1}=\frac{1}{2}(\mathbf{u}, \mathbf{u})_{E}-\mathcal{W}(u)+\frac{1}{2} \int_{\partial V_{3}} k \mathbf{u}^{2} d S+\frac{1}{2} \int_{\partial V_{4}} k^{\prime} u_{n}^{2} d S .
$$

Denoting

$$
(\mathbf{u}, \mathbf{v})_{E_{1}}=(\mathbf{u}, \mathbf{v})_{E}+\int_{\partial V_{3}} k \mathbf{u} \cdot \mathbf{v} d S+\int_{\partial V_{4}} k^{\prime} u_{n} v_{n} d S, \quad v_{n}=\mathbf{v} \cdot \mathbf{n}
$$

we get

$$
\mathcal{E}_{1}(u)=\frac{1}{2}(\mathbf{u}, \mathbf{u})_{E_{1}}-\mathcal{W},
$$

which shares the same form as $\mathcal{E}(u)$. Since the terms that complement $(\mathbf{u}, \mathbf{u})_{E}$ to $(\mathbf{u}, \mathbf{u})_{E_{1}}$ are positive, from (3), we immediately get

$$
\|\mathbf{u}\|_{W} \leq c_{2}\|\mathbf{u}\|_{E_{1}}, \quad\|\mathbf{u}\|_{E_{1}}=(\mathbf{u}, \mathbf{u})_{E_{1}}^{1 / 2}
$$


if $\left.\mathbf{u}\right|_{\partial V_{1}}=\mathbf{0}$. Moreover, if $\partial V_{3}$ is not empty, we get the last inequality (with a different constant $c_{2}$ ), even if $\partial V_{1}$ is empty. So introducing the space $E_{1}$ as the completion of the set of vector functions $\mathbf{u} \in\left(C^{(1)}(\bar{V})\right)^{3}$ satisfying (1), and practically repeating the proof of Theorem 2.1 , we get the following.

Theorem 2.4. Let the Cartesian components $\left(F_{1}, F_{2}, F_{3}\right)$ of $\mathbf{F}$ belong to the space $L^{6 / 5}(V)$ and the Cartesian components $\left(f_{1}, f_{2}, f_{3}\right)$ of $\mathbf{f}$ belong to $L^{4 / 3}\left(\partial V_{2}\right)$. There exists a weak solution $\mathbf{u} \in E_{1}$ to the equilibrium problem for an elastic body with elastic support, which for any $\mathbf{v} \in E_{1}$ satisfies the equation

$$
(\mathbf{u}, \mathbf{v})_{E_{1}}-\int_{V} \mathbf{F} \cdot \mathbf{v} d V-\int_{\partial V} \mathbf{f} \cdot \mathbf{v} d S=0
$$

Furthermore, $\mathbf{u}$ is unique.

If $\partial V_{1}$ and $\partial V_{3}$ are empty, the form of the corresponding existence theorem depends on the properties of the foundation over $\partial V_{4}$. If $\|\mathbf{u}\|_{F}=\left(\int_{\partial V_{4}} k^{\prime} u_{n}^{2} d S\right)^{1 / 2}$ is a norm on the set of rigid displacements $\mathbf{u}_{r}$ (it suffices to check that $\left\|\mathbf{u}_{r}\right\|_{F}=0$ implies $\left.\mathbf{u}_{r}=\mathbf{0}\right)$, then the norms $\|\cdot\|_{E_{1}}$ and $\|\cdot\|_{W}$ are equivalent on $\left(W^{1,2}(V)\right)^{3}$ and the last theorem continues to hold. But if the equation $\left\|\mathbf{u}_{r}\right\|_{F}=0$ has a nontrivial solution, i.e. if there are free rigid displacements of the body, then in the theorem, we should require that the load be self-balanced with respect to the corresponding rigid motions of the body.

\subsection{An elastic body with surface tension}

Existence theorems for the class of equilibrium problems pertaining to elastic bodies with surface tensions can be established for problems describing elastic bodies with elastic support. Such problems arise in the theory of nanomaterials. Clearly, points on the surface of an elastic body behave differently from the internal points, and hence accumulate additional energy under deformation. For the average-sized bodies used in traditional engineering, the additional surface strain energy is small enough to neglect. This is not the case for very small bodies, however. Here, we consider only the case in which the surface action is modeled as a thin flexible film attached to the body surface that accumulates additional strain energy due to tangential deformation. The displacements of points of the film coincide with the surface displacements of the body. In the tangential plane, the two-dimensional (2D) stress tensor $\tau=\left(\tau^{i j}\right)$ is related to the components of the strain tensor $\epsilon_{k l}$ for the film by Hooke's law $\tau^{i j}=c_{S}^{i j k l} \epsilon_{k l}$, where the tensor of elastic moduli $\left(c_{S}^{i j k l}\right)$ possesses the usual properties of the elastic moduli tensor of 2D elasticity. Hence, the strain energy density $U=\frac{1}{2} c_{S}^{i j k l} \epsilon_{i j} \epsilon_{k l}$ is positive definite.

Being deformed, the film is characterized by a stress vector $\mathbf{t}_{S}$ that should be added to the usual stress vector $\mathbf{t}$ on the boundary. So on $\partial V$, we get the stress boundary condition $\mathbf{t}=\mathbf{f}+\mathbf{t}_{S}$. Starting with the definition of a solution to the problem as a minimizer of the total potential energy functional and assuming the solution to be sufficiently smooth, by standard methods in the calculus of variations, we can show that $\mathbf{t}_{S}=\nabla_{S} \boldsymbol{\tau}$, where $\nabla_{S}=\nabla-\mathbf{n} \partial / \partial z$, the $2 \mathrm{D}$ gradient over the tangential plane, and $z$ is the coordinate along $\mathbf{n}$, the external unit normal to $\partial V$.

For this problem, the total potential energy functional takes the form

$$
\mathcal{E}_{S}(\mathbf{u})=\mathcal{E}(\mathbf{u})+\mathcal{U}(\mathbf{u})-\mathcal{W}(\mathbf{u}), \quad \mathcal{U}(\mathbf{u})=\int_{\partial V} U(\mathbf{u}) d S
$$

Supposing a minimum point of $\mathcal{E}_{S}(\mathbf{u})$ on the set of sufficiently smooth vector functions that vanish on $\partial V_{1}$, we get the displacement field that describes the equilibrium state for a body with surface tension. This displacement field makes the first variation of $\mathcal{E}_{S}(\mathbf{u})$ zero for all admissible virtual displacements. Again, using this equality for the first variation of $\mathcal{E}_{S}(\mathbf{u})$, we can introduce the definition of a weak (energy) solution to the problem. We first introduce the energy inner product

$$
(\mathbf{u}, \mathbf{v})_{E S}=(\mathbf{u}, \mathbf{v})_{E}+(\mathbf{u}, \mathbf{v})_{S}, \quad(\mathbf{u}, \mathbf{v})_{S}=\int_{\partial V} c_{S}^{i j k l} \epsilon_{k l}(\mathbf{u}) \epsilon_{i j}(\mathbf{v}) d S
$$

and the energy space $E_{S}$ as the completion, with respect to the corresponding induced norm $\|\cdot\|_{E S}$, of the subspace consisting of those vector functions in $C^{(1)}(\bar{V})$ that vanish on $\partial V_{1}$. 
An energy (weak) solution of the equilibrium problem for an elastic body with surface stresses is an element $\mathbf{u} \in E_{S}$ that satisfies the equation

$$
(\mathbf{u}, \mathbf{v})_{E S}-\mathcal{W}(\mathbf{v})=0
$$

for any $\mathbf{v} \in E_{S}$. Note that the parts of the surface integrals over $\partial V_{1}$ are zero, so we use integration over $\partial V$ as well as in the later formulation of the existence theorem.

As earlier, to formulate an existence-uniqueness theorem for the problem, we should establish that $\mathcal{W}(\mathbf{v})$ is a bounded linear functional in $E_{S}$. By the structure of the inner products in $E_{0}$ and $E_{S}$, we see that the elements of $E_{S}$ also belong to $E_{0}$, and so for boundedness of $\mathcal{W}(\mathbf{v})$, we could use the conditions of Theorem 2.1. However, by the $2 \mathrm{D}$ version of Korn's inequality, the additional term in $(\cdot, \cdot)_{E S}$ shows that the elements of $E_{S}$ also belong to $\left(W^{1,2}(\partial V)\right)^{3}$, and hence we can take weaker conditions for the tangential surface load on $\partial V$. So we can formulate the following.

Theorem 2.5. Let the Cartesian components $\left(F_{1}, F_{2}, F_{3}\right)$ of $\mathbf{F}$ belong to $L^{6 / 5}(V)$. Furthermore, let $f_{n} \in$ $L^{4 / 3}\left(\partial V_{2}\right)$, where $f_{n}$ is the projection of $\mathbf{f}$ on $\mathbf{n}$, and let the projection of $\mathbf{f}$ onto the tangential plane belong to $\left(L^{\infty}(\partial V)\right)^{2}$. There exists a unique energy (weak) solution $\mathbf{u} \in E_{S}$ to the equilibrium problem for an elastic body with surface stresses, satisfying equation (5) for any $\mathbf{v} \in E_{S}$.

In a similar way, we can formulate an existence-uniqueness theorem for a free bounded elastic body with surface stresses under a self-balanced load. We can also consider the model of an elastic body with surface stresses when some part of the boundary is elastically supported, or when we take another model of the surface film that offers bending resistance.

\section{Vibration problems}

In similar fashion, we may consider the problem of vibration for various models of a bounded elastic body. Now the weak setup of the problem for an elastic body occupying volume $V$ and clamped over $\partial V_{1}$ is formulated as follows.

Problem. Find $\mathbf{u} \in E_{0}$ and a parameter $\omega$, called an eigen-pair of the problem, such that $\|\mathbf{u}\|_{E} \neq 0$ identically and for each $\mathbf{v} \in E_{0}$, the equation

$$
(\mathbf{u}, \mathbf{v})_{E}=\omega^{2} \int_{V} \rho \mathbf{u} \cdot \mathbf{v} d V
$$

holds. Assume the density $\rho$ of the material is a positive, piecewise continuous function.

If $\mathbf{u} \in E_{0}$, then by the properties of $E_{0}$, we get

$$
\left|\int_{V} \rho \mathbf{u} \cdot \mathbf{v} d V\right| \leq c_{6}\|\mathbf{u}\|_{E}\|\mathbf{v}\|_{E}
$$

So $\int_{V} \rho \mathbf{u} \cdot \mathbf{v} d V$ is a bounded linear functional with respect to $\mathbf{v} \in E_{0}$. Applying the Riesz representation theorem, we get

$$
\int_{V} \rho \mathbf{u} \cdot \mathbf{v} d V=(\mathbf{v}, A \mathbf{u})_{E},
$$

where $A$ is an operator in $E_{0}$. In Lebedev and Vorovich [17], it is shown that $A$ is a bounded selfadjoint operator. Moreover, it is compact and positive definite if $\rho$ is piecewise continuous and satisfies $\rho>\rho_{0}>0$, where $\rho_{0}$ is a constant, which we will assume from now on. We will also use the inner product in $\left(L^{2}(V)\right)^{3}$ in the form

$$
(\mathbf{u}, \mathbf{v})_{\left(L^{2}(V)\right)^{3}}=\int_{V} \rho \mathbf{u} \cdot \mathbf{v} d V
$$

which induces the norm $\|\mathbf{u}\|_{\left(L^{2}(V)\right)^{3}}=\left((\mathbf{u}, \mathbf{u})_{\left(L^{2}(V)\right)^{3}}\right)^{1 / 2}$ equivalent to the standard norm of $\left(L^{2}(V)\right)^{3}$ when $\rho>0$ is piecewise continuous.

In Lebedev and Vorovich [17] (Theorem 2.14.2), it is shown that this implies the following.

Theorem 3.1. Suppose an elastic body occupies a bounded volume $V$ in $\mathbb{R}^{3}$ with boundary satisfying the cone property. Then, the following statements hold. 
- All the eigenfrequencies $\omega_{k}$ are positive, and the set of all $\left(\omega_{k}\right)$ is countable and has no finite accumulation point.

- $\quad$ To each $\omega_{k}$, there corresponds no more than a finite set of linearly independent eigenmodes $\mathbf{u}_{k i}$ from which we can construct a set of the same number of elements that are orthogonal in $E_{0}$ and $\left(L^{2}(V)\right)^{3}$.

- $\quad$ The set of all eigenmodes $\left(\mathbf{u}_{k i}\right)$ is an orthonormal basis of $E_{0}$. Moreover, $\left(\mathbf{u}_{k i}\right)$ is an orthogonal basis in $\left(L^{2}(V)\right)^{3}:\left(\mathbf{u}_{k}, \mathbf{u}_{m}\right)_{\left(L^{2}(V)\right)^{3}}=0$ for $k \neq m$.

We also mention that the pairs $\left(\mathbf{u}_{k}, \omega_{k}\right)$ can be found by the standard procedure of minimizing Rayleigh's quotient over the subspace of $E_{0}$ that is orthogonal to the first $k-1$ eigenmodes.

We can establish a similar theorem for the properties of the spectrum of a free bounded body. The first eigenfrequency is now $\omega=0$. To this, there corresponds a set of eigenmodes of the form $\mathbf{a}+\mathbf{b} \times \mathbf{r}$ that is six dimensional. The other eigenmodes and eigenfrequencies should be found on the space $E_{f}$ that differs from $E_{f}$ by the condition (8): the elements of $E_{f \rho}$ should satisfy

$$
\int_{V} \rho \mathbf{u} d V=\mathbf{0}, \quad \int_{\mathbf{V}} \rho \mathbf{r} \times \mathbf{u} \mathbf{d} \mathbf{V}=\mathbf{0} .
$$

The space $E_{f \rho}$ has properties similar to those of $E_{f}$. The corresponding theorem for a free elastic body mimics Theorem 3.1, with $E_{0}$ replaced by $E_{f \rho}$.

We may extend Theorem 3.1 to the other problems we have considered. Formally, we need only change the space $E_{0}$ to the energy space of the corresponding problem. The operator $A$ will also change, as its definition hinges on the corresponding energy inner product. For vibration problems for free bodies, the formulation of the theorem changes a bit as there appears $\omega_{0}=0$, which corresponds to six degrees of freedom of the body.

\section{Dynamic problems}

Adding to the volume forces $\mathbf{F}$ the inertia forces $-\rho \ddot{\mathbf{u}}$, which changes $\mathbf{F}$ to $\mathbf{F}-\rho \ddot{\mathbf{u}}$, we formally obtain the equations of the corresponding dynamic problems for all the elastic models we have considered. Here, an overdot denotes the partial derivative with respect to time $t$ so that $\dot{\mathbf{u}}=\partial \mathbf{u} / \partial t$ and $\ddot{\mathbf{u}}=\partial^{2} \mathbf{u} / \partial t^{2}$. Everything we will establish for problems of classical elasticity can be easily transferred to the other models of this paper: one should change formally the energy space for an elastic body to the energy space of the corresponding problem, and change in the equations $(\cdot, \cdot)_{E}$ to the notation of the corresponding inner product. So we will not formulate the results for other elastic models.

The dynamic equation for elasticity, based on (5), takes the form

$$
-\int_{V} \rho \ddot{\mathbf{u}} \cdot \mathbf{v} d V=(\mathbf{u}, \mathbf{v})_{E}-\int_{V} \mathbf{F} \cdot \mathbf{v} d V-\int_{\partial V_{2}} \mathbf{f} \cdot \mathbf{v} d S
$$

where the vector functions $\mathbf{u}, \mathbf{v}$ now depend on $t$ and $\mathbf{r}$. This equation for the unknown displacement field $\mathbf{u}$ must hold for all admissible $\mathbf{v}$.

First we consider the problem when $\mathbf{u}$ (and hence $\mathbf{v}$ ) is zero on $\partial V_{1}$, whereas over the remainder $\partial V_{2}$ of $\partial V$, the stress vector is given as f. To complete the formulation of the problem, we supplement equation (11) and the boundary conditions with the initial conditions

$$
\left.\mathbf{u}\right|_{t=0}=\tilde{\mathbf{u}}_{0},\left.\quad \dot{\mathbf{u}}\right|_{t=0}=\tilde{\mathbf{u}}_{1} .
$$

Now let us change (11) to the form needed for the weak setup. We integrate both sides of (11) with respect to $t$ over $[0, T]$, where $T$ is some arbitrary but fixed instant, and then integrate by parts with respect to $t$ in the integral that contains the term $\ddot{\mathbf{u}}$

$$
\int_{0}^{T} \int_{V} \rho \dot{\mathbf{u}} \cdot \dot{\mathbf{v}} d V d t-\left.\int_{V} \rho \dot{\mathbf{u}} \cdot \mathbf{v} d V\right|_{0} ^{T}=\int_{0}^{T}(\mathbf{u}, \mathbf{v})_{E} d t-\int_{0}^{T} \mathcal{W}(\mathbf{v}) d t .
$$

Now we restrict the set of admissible $\dot{\mathbf{v}}$ to those that are zero when $t=T$ and change the value of $\mathbf{u}$ at $t=0$ to its initial value

$$
\int_{0}^{T} \int_{V} \rho \dot{\mathbf{u}} \cdot \dot{\mathbf{v}} d V d t+\left.\int_{V} \rho \tilde{\mathbf{u}}_{1} \cdot \mathbf{v} d V\right|_{t=0}=\int_{0}^{T}(\mathbf{u}, \mathbf{v})_{E} d t-\int_{0}^{T} \mathcal{W}(\mathbf{v}) d t
$$


Note that, assuming the existence of a sufficiently smooth solution to (13), and using the traditional tools of the calculus of variations, we can derive the equations of motion for the elastic body, the load conditions over $\partial V_{2}$, and the initial condition for $\dot{\mathbf{u}}$. So (13), which holds for all admissible $\mathbf{v}$, together with the zero restriction for $\mathbf{u}$ on $\partial V_{1}$ and the initial condition for $\mathbf{u}$, constitute a setup for the dynamic problem of linear elasticity in displacements. So we use (13) as a basis for introducing the weak setup of the dynamic problem. Next, we wish to underline that, starting with the initial work by O.A. Ladyzhenskaya, the weak setup of hyperbolic equations was considered for Dirichlet's boundary condition, that is, when $\left.\mathbf{u}\right|_{\partial V}=0$. For elastic problems, this excluded the case of given forces $\mathbf{f}$ on the boundary. However, such forces are more important in engineering than the body forces $\mathbf{F}$, as the class of the existing forces $\mathbf{F}$ is quite restrictive. The reason for such elimination of $\mathbf{f}$ is that $\mathbf{F}$ can be estimated using the kinetic energy, whereas for estimating $\mathbf{f}$, it is necessary to consider the strain energy, which is defined in mechanics but not for general hyperbolic equations.

Let us introduce the space in which we will seek a weak solution to the dynamic problem. Let $C_{1}$ be the set of vector functions $\mathbf{u}$ that are twice continuously differentiable on $Q_{T}=[0, T] \times \bar{V}$, with values in $\mathbb{R}^{3}$ that vanish on $\partial V_{1}$. Introduce on $C_{1}$ the inner product

$$
(\mathbf{u}, \mathbf{v})_{\mathcal{E}_{T}}=\int_{0}^{T}\left(\int_{V} \rho \dot{\mathbf{u}} \cdot \dot{\mathbf{v}} d V+(\mathbf{u}, \mathbf{v})_{E}\right) d t .
$$

The completion of $C_{1}$ with respect to the corresponding norm $\|\cdot\|_{\mathcal{E}_{T}}$ is called the energy space $\mathcal{E}_{T}$. Similar to the fact that $E_{0}$ is a subspace of $\left(W^{1,2}(V)\right)^{3}$, we can easily establish that $\mathcal{E}_{T}$ is a subspace of $\left(W^{1,2}\left(Q_{T}\right)\right)^{3}$ and that on $\mathcal{E}_{T}$ the norm $\|\cdot\|_{\mathcal{E}_{T}}$ is equivalent to the norm of $\left(W^{1,2}\left(Q_{T}\right)\right)^{3}$. To introduce the definition of weak solution to the problem, we also define a subspace $\mathcal{E}_{T}^{\circ}$ of $\mathcal{E}_{T}$ that is the completion of the subset of $C_{1}$ consisting of the vector functions vanishing when $t=T$.

Definition 4.1. $\mathbf{u} \in \mathcal{E}_{T}$ is a weak solution to the dynamic problem of elasticity if it satisfies (13) for any $\mathbf{v} \in \mathcal{E}_{T}^{\circ}$ and $\mathbf{u}(0, \mathbf{r})=\tilde{\mathbf{u}}_{0}(\mathbf{r})$ in the sense of $L^{2}(V)$.

First we introduce a few Banach spaces for the functions $x=x(t)$ of a parameter $t$, taking the values in a Banach space $X$. By $L^{p}(0, T ; X), p \geq 1$, we denote a Banach space of functions $x(t)$ on the segment $t \in[a, b]$ with the norm

$$
\|x\|_{L^{p}(a, b ; X)}=\left(\int_{a}^{b}\|x(t)\|_{X}^{p} d t\right)^{1 / p} .
$$

Similarly, $C(a, b ; X)$ is a Banach space of functions $x(t)$ of the parameter $t \in[a, b]$, taking values in $X$ that are continuous on $[a, b]$. The norm in $C(a, b ; X)$ is

$$
\|x\|_{C(a, b ; X)}=\max _{[a, b]}\|x(t)\|_{X} .
$$

We also introduce the completion of the set of functions $x(t)$, taking values in $X$ and such that $x(t)$ and $\dot{x}(t)$ are in $C(0, T ; X)$, with respect the norm

$$
\|x\|_{W^{1,2}(a, b ; X)}=\left(\int_{a}^{b}\left(\|\dot{x}(t)\|_{X}^{2}+\|x(t)\|_{X}^{2}\right) d t\right)^{1 / 2},
$$

denoted by $W^{1,2}(a, b ; X)$. It is known that $W^{1,2}(a, b ; X)$ is continuously imbedded to $C(a, b ; X)$ provided $a, b$ are finite.

For the initial data, we assume that

$$
\tilde{\mathbf{u}}_{0} \in E_{0}, \quad \tilde{\mathbf{u}}_{1} \in\left(L^{2}(V)\right)^{3},
$$

and for the external load

$$
\mathbf{F} \in\left(L^{2}(V)\right)^{3}, \quad \mathbf{f} \in W^{1,2}\left(0, T ;\left(L^{4 / 3}\left(\partial V_{2}\right)^{3}\right) .\right.
$$

Under the conditions (14) and (15), Definition 4.1 makes sense. Indeed, for all terms of the equation except the one involving $\mathbf{f}$, the verification is routine. For the latter, we recall that by Sobolev's imbedding theorem, $W^{1,2}(V)$ 
is continuously imbedded to $L^{4}\left(\partial V_{2}\right)$, and so for $\mathbf{v} \in \mathcal{E}_{T}$, we see that $\mathbf{v}$ is an element of $L^{2}\left(0, T ;\left(L^{4}\left(\partial V_{2}\right)\right)^{3}\right)$. Then

$$
\begin{aligned}
\left|\int_{0}^{T} \int_{\partial V_{2}} \mathbf{f} \cdot \mathbf{v} d S d t\right| & \leq \int_{0}^{T}\left(\int_{0}^{T} \int_{\partial V_{2}}|\mathbf{f}|^{4 / 3} d S\right)^{3 / 4}\left(\int_{\partial V_{2}}|\mathbf{v}|^{4} d S\right)^{1 / 4} d t \\
& \leq m\|\mathbf{f}\|_{L^{2}\left(0, T ;\left(L^{4 / 3}\left(\partial V_{2}\right)\right)^{3}\right)}\|\mathbf{v}\|_{\mathcal{E}_{T}}
\end{aligned}
$$

with a constant $m$ independent of $\mathbf{v}$. Hence, this term in the definition also makes sense. Now we formulate the following.

Theorem 4.1. Suppose the conditions (14) and (15) hold. There exists $\mathbf{u} \in \mathcal{E}_{T}$, a weak solution to the dynamic problem of elasticity in the sense of Definition 4.1. The solution is unique.

Uniqueness of a weak solution to the problem is well known for any boundary conditions. We will prove its existence using the Faedo-Galerkin method, which is called the Bubnov-Galerkin method in Russia.

By Theorem 3.1, there exists a set $\left\{\mathbf{u}_{k}\right\}$ that is an orthogonal basis in $E_{0}$ and in $\left(L^{2}(V)\right)^{3}$ simultaneously. Let us normalize this set in $\left(L^{2}(V)\right)^{3}$, using $\mathbf{w}_{k}=\mathbf{u}_{k} /\left\|\mathbf{u}_{k}\right\|_{\left(L^{2}(V)\right)^{3}}$ to obtain an orthonormal basis $\left\{\mathbf{w}_{k}\right\}$ in $\left(L^{2}(V)\right)^{3}$. That is, we have $\left(\mathbf{w}_{k}, \mathbf{w}_{m}\right)=\delta_{k m}$ and the set $\left\{\mathbf{w}_{k}\right\}$ is still an orthogonal basis in $E_{0}$. Henceforth, we denote the $n$th approximation of the Faedo-Galerkin method by $\mathbf{u}_{n}(t, \mathbf{r})=\sum_{k=1}^{n} c_{k}(t) \mathbf{w}_{k}(\mathbf{r})$. To get the equations of the method, we substitute $\mathbf{u}_{n}$ into (11); as $\mathbf{v}$, we take successively $\mathbf{w}_{k}$ for each $k=1, \ldots, n$, getting $n$ linear equations

$$
-\int_{V} \rho \ddot{\mathbf{u}}_{n} \cdot \mathbf{w}_{k} d V=\left(\mathbf{u}_{n}, \mathbf{w}_{k}\right)_{E}-\int_{V} \mathbf{F} \cdot \mathbf{w}_{k} d V-\int_{\partial V_{2}} \mathbf{f} \cdot \mathbf{w}_{k} d S .
$$

By the orthogonality properties of $\left\{\mathbf{w}_{k}\right\}$, we get the following simultaneous equations in terms of unknown functions $c_{k}(t)$

$$
\ddot{c}_{k}(t)+c_{k}(t)\left(\mathbf{w}_{k}, \mathbf{w}_{k}\right)_{E}=\int_{V} \mathbf{F} \cdot \mathbf{w}_{k} d V+\int_{\partial V_{2}} \mathbf{f} \cdot \mathbf{w}_{k} d S, \quad k=1, \ldots, n .
$$

These turn out to be $n$ independent equations, for each of which the initial data (12) define the following initial conditions for $c_{k}$

$$
c_{k}(0)=\frac{\left(\tilde{\mathbf{u}}_{0}, \mathbf{w}_{k}\right)_{E}}{\left\|\mathbf{w}_{k}\right\|_{E}}, \quad \dot{c}_{k}(0)=\left(\tilde{\mathbf{u}}_{1}, \mathbf{w}_{k}\right)_{L^{2}(V)} .
$$

Each equation of (18) is a second-order ordinary differential equation with constant coefficients. By the conditions of the theorem, its right-hand side belongs to $L(0, T)$. Indeed, for the first integral term, this is evident as it belongs to $L^{2}(0, T)$. Using a chain of inequalities similar to (16), we can also show that as a function, the term involving $\mathbf{F}$ belongs to $L^{2}(0, T)$ and hence $L(0, T)$. Similarly, we can show that the term involving $\mathbf{f}$ lies in $L(0, T)$. Hence, on $[0, T]$, there exists a unique solution $\left(c_{1}(t), \ldots, c_{n}(t)\right)$ of the system, with $c_{k}^{\prime \prime}(t) \in L(0, T)$. So all $c_{k}(t)$ and $c_{k}^{\prime}(t)$ are continuous on $[0, T]$.

The next step of the proof is to establish a priori estimates for the $n$th Faedo-Galerkin approximation, $\mathbf{u}_{n}(t)=\sum_{k=1}^{n} c_{k}(t) \mathbf{w}_{k}(\mathbf{r})$, defined by (18) and (19). We multiply (17) by $\dot{c}_{k}(t)$ and sum over $k$, obtaining

$$
\int_{V} \rho \ddot{\mathbf{u}}_{n} \cdot \dot{\mathbf{u}}_{n} d V+\left(\mathbf{u}, \dot{\mathbf{u}}_{n}\right)_{E}=\int_{V} \mathbf{F} \cdot \dot{\mathbf{u}}_{n} d V+\int_{\partial V_{2}} \mathbf{f} \cdot \dot{\mathbf{u}}_{n} d S .
$$

This can be rewritten as

$$
\frac{d}{d t}\left(\frac{1}{2} \int_{V} \rho \dot{\mathbf{u}}_{n} \cdot \dot{\mathbf{u}}_{n} d V+\frac{1}{2}\left(\mathbf{u}_{n}, \mathbf{u}_{n}\right)_{E}\right)=\int_{V} \mathbf{F} \cdot \dot{\mathbf{u}}_{n} d V+\int_{\partial V_{2}} \mathbf{f} \cdot \dot{\mathbf{u}}_{n} d S .
$$

Changing $t$ to $\tau$, multiplying the equality by 2 , and then integrating it with respect to $\tau$ over $[0, t]$, we get

$$
\begin{aligned}
\left(\int_{V} \rho \dot{\mathbf{u}}_{n} \cdot \dot{\mathbf{u}}_{n} d V\right. & \left.+\left(\mathbf{u}, \mathbf{u}_{n}\right)_{E}\right)\left.\right|_{\tau=t}=\left.\left(\int_{V} \rho \dot{\mathbf{u}}_{n} \cdot \dot{\mathbf{u}}_{n} d V+\left(\mathbf{u}_{n}, \mathbf{u}_{n}\right)_{E}\right)\right|_{\tau=0} \\
& +2 \int_{0}^{t}\left(\int_{V} \mathbf{F} \cdot \dot{\mathbf{u}}_{n} d V+\int_{\partial V_{2}} \mathbf{f} \cdot \dot{\mathbf{u}}_{n} d S\right) d \tau
\end{aligned}
$$


Let us estimate the terms on the right-hand side of (20). Using the orthogonality properties of $\left\{\mathbf{w}_{k}\right\}$, we get

$$
\left.\left(\int_{V} \rho \dot{\mathbf{u}}_{n} \cdot \dot{\mathbf{u}}_{n} d V+\left(\mathbf{u}_{n}, \mathbf{u}_{n}\right)_{E}\right)\right|_{\tau=0} \leq\left(\left(\tilde{\mathbf{u}}_{1}, \tilde{\mathbf{u}}_{1}\right)_{L^{2}(V)}+\left(\tilde{\mathbf{u}}_{0}, \tilde{\mathbf{u}}_{0}\right)_{E}\right) \equiv m_{1} .
$$

Here, by $m_{k}$, we will denote the constants that do not depend on $n$ and $t$. By Schwarz's inequality,

$$
\begin{aligned}
2\left|\int_{0}^{t} \int_{V} \mathbf{F} \cdot \dot{\mathbf{u}}_{n} d V d \tau\right| & \leq 2\left(\int_{0}^{t}\|\mathbf{F}\|_{\left(L^{2}(V)\right)^{3}}^{2} d \tau\right)^{1 / 2}\left(\int_{0}^{t}\left\|\dot{\mathbf{u}}_{n}\right\|_{\left(L^{2}(V)\right)^{3}}^{2} d \tau\right)^{1 / 2} \\
& \leq m_{2}+\int_{0}^{t}\left\|\dot{\mathbf{u}}_{n}\right\|_{\left(L^{2}(V)\right)^{3}}^{2} d \tau
\end{aligned}
$$

Now we consider the term containing $\mathbf{f}$. Integrating by parts, we get

$$
\left|\int_{0}^{t} \int_{\partial V_{2}} \mathbf{f} \cdot \dot{\mathbf{u}}_{n} d S\right|=\left|-\int_{0}^{t} \int_{\partial V_{2}} \dot{\mathbf{f}} \cdot \mathbf{u}_{n} d S d \tau+\int_{\partial V_{2}} \mathbf{f} \cdot \mathbf{u}_{n} d S\right|_{\tau=0}^{\tau=t} \mid .
$$

For the first integral on the right, we get successively

$$
\begin{aligned}
\left|-\int_{0}^{t} \int_{\partial V_{2}} \dot{\mathbf{f}} \cdot \mathbf{u}_{n} d S d \tau\right| & \leq \int_{0}^{t}\|\mathbf{f}\|_{\left(L^{4 / 3}\left(\partial V_{2}\right)\right)^{3}}\left\|\mathbf{u}_{n}\right\|_{\left(L^{4}\left(\partial V_{2}\right)\right)^{3}} d \tau \\
& \leq\|\mathbf{f}\|_{L^{2}\left(0, T ;\left(L^{4 / 3}\left(\partial V_{2}\right)\right)^{3}\right.}\left\|\mathbf{u}_{n}\right\|_{L^{2}\left(0, t ;\left(L^{4}\left(\partial V_{2}\right)\right)^{3}\right)} \\
& \leq m_{3}+m_{4} \int_{0}^{t}\left\|\mathbf{u}_{n}\right\|_{E}^{2} d \tau
\end{aligned}
$$

Using the elementary inequality $|a b| \leq a^{2} /(2 c)+c b^{2}, c>0$, and the established properties for $\mathbf{f}, \mathbf{u}_{n}$, we have for the second term

$$
\begin{aligned}
\left|\int_{\partial V_{2}} \mathbf{f} \cdot \mathbf{u}_{n} d S\right|_{\tau=0}^{\tau=t} \mid & \leq\left.\left|\int_{\partial V_{2}} \mathbf{f} \cdot \mathbf{u}_{n} d S\right|_{\tau=t}|+| \int_{\partial V_{2}} \mathbf{f} \cdot \mathbf{u}_{n} d S\right|_{\tau=0} \mid \\
& \leq\left.\|\mathbf{f}\|_{\left(L^{4 / 3}\left(\partial V_{2}\right)\right)^{3}}\left\|\mathbf{u}_{n}\right\|_{\left(L^{4}\left(\partial V_{2}\right)\right)^{3}}\right|_{\tau=t}+m_{5} \\
& \leq m_{6}+\left.\frac{1}{2}\left\|\mathbf{u}_{n}\right\|_{E}^{2}\right|_{\tau=t}
\end{aligned}
$$

Using (21)-(25), from the equality (20), we deduce

$$
\left.\left(\int_{V} \rho \dot{\mathbf{u}}_{n} \cdot \dot{\mathbf{u}}_{n} d V+\left(\mathbf{u}, \mathbf{u}_{n}\right)_{E}\right)\right|_{\tau=t} \leq m_{7}+m_{8} \int_{0}^{t}\left(\int_{V} \rho \dot{\mathbf{u}}_{n} \cdot \dot{\mathbf{u}}_{n} d V+\left(\mathbf{u}, \mathbf{u}_{n}\right)_{E}\right) d \tau
$$

for all $0<t \leq T$.

By Gronwall's inequality, from (26), we get the estimate

$$
\left.\left(\int_{V} \rho \dot{\mathbf{u}}_{n} \cdot \dot{\mathbf{u}}_{n} d V+\left(\mathbf{u}, \mathbf{u}_{n}\right)_{E}\right)\right|_{\tau=t} \leq m_{9} \quad \text { for all } 0<t \leq T,
$$

where the constant $m_{9}$ is independent of $n$. From this, we also deduce that

$$
\left\|\mathbf{u}_{n}\right\|_{\mathcal{E}_{T}} \leq m_{9} T
$$

Now the proof of existence of a weak solution is quite traditional. From the boundedness of the sequence $\left\{\mathbf{u}_{n}\right\}$ in $\mathcal{E}_{T}$, we know that it contains a weakly Cauchy subsequence $\left\{\mathbf{u}_{n_{i}}\right\}$ in $\mathcal{E}_{T}$. The weak limit w- $\lim _{n_{i} \rightarrow \infty} \mathbf{u}_{n_{i}}=\mathbf{u}^{*}$ is a 
weak solution to the problem under consideration. Indeed, we multiply the $k$ th equation of (19) by a continuously differentiable function $d_{k}(t)$ such that $d_{k}(T)=0$, sum the equations over $k$, and integrate the result over $[0, T]$. Integrating then by parts with respect to $t$, and denoting $\hat{\mathbf{v}}=\sum_{k=1}^{n} d_{k}(t) \mathbf{w}_{k}(\mathbf{r})$, we get an equation that looks quite similar to (13), participating in the definition of the weak solution

$$
\int_{0}^{T}\left(\int_{V} \rho \dot{\mathbf{u}}_{n} \cdot \dot{\hat{\mathbf{v}}} d V-\left(\mathbf{u}_{n}, \hat{\mathbf{v}}\right)_{E}\right) d t=-\left.\int_{V} \rho \dot{\mathbf{u}}_{n} \cdot \hat{\mathbf{v}} d V\right|_{t=0}-\int_{0}^{T} \mathcal{W}(\hat{\mathbf{v}}) d t .
$$

Now we fix $\hat{\mathbf{v}}$, take as $\mathbf{u}_{n}$ only $\mathbf{u}_{n_{i}}$, and then take the limit in the equality as $n_{i} \rightarrow \infty$. As $\mathbf{u}_{n_{i}}$ converges weakly to $\mathbf{u}^{*}$, and $\left.\mathbf{u}_{n_{i}}\right|_{t=0}$ converges strongly to $\tilde{\mathbf{u}}_{1}$ in $E_{0}$, we get

$$
\int_{0}^{T}\left(\int_{V} \rho \dot{\mathbf{u}}^{*} \cdot \dot{\hat{\mathbf{v}}} d V-\left(\mathbf{u}^{*}, \hat{\mathbf{v}}\right)_{E}\right) d t=-\left.\int_{V} \rho \tilde{\mathbf{u}}_{1} \cdot \hat{\mathbf{v}} d V\right|_{t=0}-\int_{0}^{T} \mathcal{W}(\hat{\mathbf{v}}) d t
$$

which is the equation (13) for $\mathbf{u}^{*}$ when $\mathbf{v}=\hat{\mathbf{v}}$. As the set of all such $\hat{\mathbf{v}}$ is dense in $\mathcal{E}_{T}^{\circ}$, we see that (13) for $\mathbf{u}=\mathbf{u}^{*}$ holds for any $\hat{\mathbf{v}} \in \mathcal{E}_{T}^{\circ}$, and so $\mathbf{u}^{*}$ is its solution in the weak sense. Clearly the initial condition $\left.\mathbf{u}^{*}\right|_{t=0} \tilde{\mathbf{u}}_{0}$ holds as well, and the proof of the theorem is complete.

Remarks. (1) We will not discuss the smoothness properties of the solution $\mathbf{u}^{*}$. Indeed, it can be shown that $\mathbf{u}^{*}$ is smoother than the theorem states $[18,19]$.

(2) By the conditions of the theorem, $\mathbf{f}$ should be continuous with respect to time, with $\mathbf{f} \in$ $C\left(0, T ;\left(L^{4 / 3}\left(\partial V_{2}\right)\right)^{3}\right.$. We can suppose that $\mathbf{f}$ has a few finite jumps on $[0, T]$ at points $t_{k}$, requiring that $\mathbf{f}$ belong to $W^{1,2}\left(t_{k}, t_{k+1} ;\left(L^{4 / 3}\left(\partial V_{2}\right)\right)^{3}\right)$ on each $\left[t_{k}, t_{k+1}\right], t_{0}=0$. For this, step by step, we should repeat the above considerations for $\mathbf{u}_{n}$ on one segment to the next, taking the final value on the previous segment as the initial value on the next segment.

(3) A technical remark. The proofs for the dynamic problems that can be found in various sources, such as Lions and Magenes [18] and Ladyzhenskaya [19], are based on the fact that the integral inequality for the solution is derived through the kinetic energy term $\int_{V} \dot{\mathbf{u}} \cdot \dot{\mathbf{u}} d V$, whereas the existence of the strain energy is ignored: the authors consider abstract problems rather than problems of mechanics. Here, we essentially use the fact that the strain energy plays an important role in the total energy of the body. Hence, we can consider problems with given forces on the boundary.

\section{I. Dynamics of a free body}

Quite similar to the dynamic problem for an elastic body clamped on some part of its boundary, we can consider the existence and uniqueness of a weak solution for a free elastic body. To get the result, we can use the considerations of Theorem 3.1 almost directly. First we should change in (13) the part $\partial V_{2}$ of the boundary to $\partial V$. The trick for reducing the proof for a free body to the previously studied problem is based on the fact that the solution for a free body splits into two parts

$$
\mathbf{u}(t, \mathbf{r})=\mathbf{a}(t)+\mathbf{b}(t) \times \mathbf{r}+\hat{\mathbf{u}},
$$

where

$$
\int_{V} \rho \hat{\mathbf{u}} d V=\mathbf{0}, \quad \int_{V} \rho \mathbf{r} \times \hat{\mathbf{u}} d V=\mathbf{0}
$$

and $\mathbf{a}+\mathbf{b} \times \mathbf{r}$, with $\mathbf{a}, \mathbf{b}$ constants, describes the displacements of the rigid body occupying volume $V$. In the proof, we should use the fact that the two parts of the solution are orthogonal in $\left(L^{2}(V)\right)^{3}$, as well as with respect to the inner product $(\cdot, \cdot)_{E}$, so the dynamic equation for them also splits into two parts. Let us introduce a formal volume load $\mathbf{F}_{0}(t)+\mathbf{r} \times \mathbf{F}_{1}(t)$, where $\mathbf{F}_{k}$ depends on $t$ and is independent of $\mathbf{r}$; it is defined by the equalities

$$
\begin{gathered}
\int_{V}\left(\mathbf{F}-\mathbf{F}_{0}-\mathbf{r} \times \mathbf{F}_{1}\right) d V+\int_{\partial V} \mathbf{f} d S=\mathbf{0}, \\
\int_{V} \mathbf{r} \times\left(\mathbf{F}-\mathbf{F}_{0}-\mathbf{r} \times \mathbf{F}_{1}\right) d V+\int_{\partial V} \mathbf{r} \times \mathbf{f} d S=\mathbf{0},
\end{gathered}
$$


for any instant $t$. For the load $\left(\hat{\mathbf{F}}=\mathbf{F}-\mathbf{F}_{0}(t)-\mathbf{r} \times \mathbf{F}_{1}(t), \mathbf{f}\right)$, the resultants of the force and moment are zero. Now for the part $\hat{\mathbf{u}}$, we get the following equation

$$
\begin{gathered}
\int_{0}^{T} \int_{V} \rho \dot{\hat{\mathbf{u}}} \cdot \dot{\mathbf{v}} d V d t+\left.\int_{V} \rho \hat{\mathbf{u}}_{1} \cdot \mathbf{v} d V\right|_{t=0}=\int_{0}^{T}(\hat{\mathbf{u}}, \mathbf{v})_{E} d t-\int_{0}^{T} \hat{\mathcal{W}}(\mathbf{v}) d t \\
\hat{\mathcal{W}}(\mathbf{v})=\int_{V} \hat{\mathbf{F}} \cdot \mathbf{v} d V+\int_{\partial V} \mathbf{f} \cdot \mathbf{v} d S,
\end{gathered}
$$

where $\mathbf{v}$ also must satisfy the relations (27) and the initial value for $\dot{\hat{\mathbf{u}}}$ is $\hat{\mathbf{u}}_{1}=\tilde{\mathbf{u}}_{1}-\dot{\mathbf{a}}(0)-\dot{\mathbf{b}}(0) \times \mathbf{r}$; constant vectors $\dot{\mathbf{a}}(0), \dot{\mathbf{b}}(0)$, the initial data for the velocity of the rigid displacements, are defined by (27)

$$
\int_{V} \rho\left(\tilde{\mathbf{u}}_{1}-\dot{\mathbf{a}}(0)-\dot{\mathbf{b}}(0) \times \mathbf{r}\right) d V=\mathbf{0}, \quad \int_{V} \rho \mathbf{r} \times\left(\tilde{\mathbf{u}}_{1}-\dot{\mathbf{a}}(0)-\dot{\mathbf{b}}(0) \times \mathbf{r}\right) d V=\mathbf{0} .
$$

Similarly, we define the initial values for $\hat{\mathbf{u}}$ and $\mathbf{a}(0), \mathbf{b}(0)$.

The equations for the rigid motion of the body are

$$
\begin{gathered}
M \ddot{\mathbf{a}}(t)+\ddot{\mathbf{b}}(t) \times \int_{V} \rho \mathbf{r} d V=\int_{V}\left(\mathbf{F}_{0}(t)+\mathbf{r} \times \mathbf{F}_{1}(t)\right) d V, \quad M=\int_{V} \rho d V, \\
\int_{V} \rho \mathbf{r} \times \ddot{\mathbf{a}} d V+\int_{V} \rho \mathbf{r} \times(\ddot{\mathbf{b}} \times \mathbf{r}) d V=\int_{V} \mathbf{r} \times\left(\mathbf{F}_{0}(t)+\mathbf{r} \times \mathbf{F}_{1}(t)\right) d V .
\end{gathered}
$$

If $\mathbf{r}=\mathbf{0}$ is the inertia center of the body, the rigid motion equations reduce to

$$
M \ddot{\mathbf{a}}(t)=\int_{V}\left(\mathbf{F}_{0}(t)+\mathbf{r} \times \mathbf{F}_{1}(t)\right) d V, \quad \mathbf{J} \cdot \ddot{\mathbf{b}}=\int_{V} \mathbf{r} \times\left(\mathbf{F}_{0}(t)+\mathbf{r} \times \mathbf{F}_{1}(t)\right) d V,
$$

where the tensor of inertia of the body $\mathbf{J}=\int_{V} \rho[(\mathbf{r} \cdot \mathbf{r}) \mathbf{I}-\mathbf{r} \otimes \mathbf{r}] d V$. The study of the solution to the initial value problem for the ordinary differential equations for $\mathbf{a}, \mathbf{b}$ is elementary.

The study of the existence and uniqueness of the part of the solution $\hat{\mathbf{u}}$ is technically similar to the studies for a solution for a body with a portion of its boundary clamped. Here, $\|\cdot\|_{E}$, on the set of functions smooth on $V$ and satisfying (27), is a norm. So we can introduce a space $E_{f}$ as the completion of the smooth vector functions satisfying (27) with respect to the norm $\|\cdot\|_{E}$ (note that it differs from the space $E_{f}$ introduced in Section 2 by the restriction of the elements that includes $\rho$, but it has the same properties). This allows us to use the same techniques of the Faedo-Galerkin method to prove existence of a weak solution. The difference with the corresponding proof of existence for the clamped body is that we should change the basis $\mathbf{w}_{k}$ to the basis that is an orthogonal set of eigenmodes of the elasticity problem with the constraints (27); it has the same properties as $\mathbf{w}_{k}$ in the space $E_{f}$ of the previous theorem. So now we can merely reformulate Theorem 3.1 as the following.

Theorem 4.2. Suppose the conditions (15) hold, with $\tilde{\mathbf{u}}_{0} \in\left(W^{1,2}(V)\right)^{3}$ and $\tilde{\mathbf{u}}_{1} \in\left(L^{2}(V)\right)^{3}$. There exists a weak solution to the dynamic problem of elasticity in the sense of Definition 4.1. The solution is unique.

Similarly, we can consider dynamic problems for other models considered in the first section. No additional formal difficulties are encountered.

\section{Conclusion}

We have considered the weak approach to the problems of equilibrium, dynamics and vibration for various linear problems arising in elasticity. By unified methods, we have considered the problems of classical linear elasticity, as well as some of their generalizations related to surface elasticity. Much attention has been given to the analysis of dynamic problems for an elastic body with a boundary that is not geometrically constrained, under the action of non-self-balanced loads; these problems have not received prior consideration in the literature. It has been shown that each time, the weak setup of the problem is well posed and therefore we can use, with justification, various numerical methods, such as the FEM, which are based on the approach. 


\section{Funding}

The first author was supported by the DFG [grant number AL 341/33-1] and by the RFBR [grant number 12-01-00038]. The second author was supported by the National University of Colombia [cod. 12955].

\section{Acknowledgements}

The authors are indebted to Michael J. Cloud for help in preparing the paper.

\section{Conflict of interest}

None declared.

\section{References}

[1] Sobolev, SL. Some applications of functional analysis in mathematical physics. $L G U$ 1950, in Russian (translated to English, AMS, 1991).

[2] Korn, A. Solution générale du problème d'équilibre dans la théorie de l'élasticité dans le cas où les efforts sont donnès à la surface. Ann Fac Sci Toulouse, Ser 2 1908; 10: 165-269.

[3] Korn, A. Über einige Ungleichungen, welche in der Theorie der elastischen und elektrischen Schwingungen eine Rolle spielen. Bull Int Cracovie Akademie Umiejet, Classe des Sci Math Nat 1909; 705-724.

[4] Gurtin, ME, and Murdoch, AI. A continuum theory of elastic material surfaces. Arch Rat Mech Analysis 1975; 57: $291-323$.

[5] Steigmann, DJ, and Ogden, RW. Elastic surface-substrate interactions. Proc R Soc Lond A 1999; 455: $437-474$.

[6] Javili, A, and Steinmann, P. On thermomechanical solids with boundary structures. Int J Solids Struct 2010; 47: 3245-3253.

[7] Duan, HL, Wang, J, and Karihaloo, BL. Theory of elasticity at the nanoscale. In: Advances in applied mechanics. Elsevier, 2008, pp. $1-68$.

[8] Wang, J, Huang, Z, Duan, H, et al. Surface stress effect in mechanics of nanostructured materials. Acta Mechanica Solida Sinica 2011; 24: 52-82.

[9] Kim, CI, Schiavone, P, and Ru, C-Q. Effect of surface elasticity on an interface crack in plane deformations. Proc R Soc A 2011; 467: 3530-3549.

[10] Kim, CI, Schiavone, P, and Ru, C-Q. A clarification of the role of crack-tip conditions in linear elasticity with surface effects. Math Mech Solids 2012. DOI: 10.1177/1081286511435227.

[11] Schiavone, P, and Ru, CQ. Solvability of boundary value problems in a theory of plane-strain elasticity with boundary reinforcement. Int J Engng Sci 2009; 47: 1331-1338.

[12] Altenbach, H, Eremeyev, VA, and Lebedev, LP. On the existence of solution in the linear elasticity with surface stresses. ZAMM 2010; 90: 231-240.

[13] Altenbach, H, Eremeyev, VA, and Lebedev, LP. On the spectrum and stiffness of an elastic body with surface stresses. ZAMM 2011; 91: 699-710.

[14] Javili, A, McBride, A, Steinmann, P, et al. Relationships between the admissible range of surface material parameters and stability of linearly elastic bodies. Phil Mag. DOI:10.1080/14786435.2012.682175.

[15] Ciarlet, P. Mathematical elasticity. Vol. I: three-dimensional elasticity. Amsterdam: North-Holland, 1988.

[16] Fichera, G. Existence theorems in elasticity. In: Flügge, S (ed.) Handbuch der physik. Berlin: Springer, 1972, pp. 347-389.

[17] Lebedev, LP, and Vorovich, II. Functional analysis in mechanics. New York: Springer-Verlag, 2003.

[18] Lions, J-L, and Magenes, E. Problems aux limites non homogènes et application, vol. 1. Paris: Dunod, 1968.

[19] Ladyzhenskaya, OA. The boundary value problems of mathematical physics. New York: Springer-Verlag, 2010. 